\title{
Mechanically Enhanced Proline Ring-Opening in Proteins
}

\author{
Jan H. Hoh \\ jan_hoh@yahoo.com
}

\begin{abstract}
Proteins such as collagen and elastin are subjected to highly repetitive stresses in the cardiovascular system. These proteins can have half-lives of many decades and, therefore, experience many tens or hundreds of millions of stress-strain cycles. Such a large number of cycles, even for a modest level of stress, subjects these molecules bond breaking and fatigue failure. To gain further insight into bond breaking in mechanically stressed proteins, the mechanical strength of bonds in the polypeptide backbone are estimated using bond dissociation energies from model compounds for individual amino acids. This analysis shows that the N-C $\alpha$ bond in proline has a bond dissociation energy that is $\sim 60 \mathrm{~kJ} / \mathrm{mol}$ less than the next weakest bond in a polypeptide backbone. Thus, when mechanically stressed, the pyrrolidine ring of proline will open before other backbone bonds break. Such mechanically enhanced proline ring-opening would maintain structural continuity of the peptide backbone while increasing the length of the backbone by $\sim 0.3 \mathrm{~nm}$ per ring-opening. The increase in length would redistribute forces to nearby molecules, thus effectively remodeling the mechanical properties of the tissue involved.
\end{abstract}

\section{Introduction}

Living organisms must cope with the forces that they generate, or that they are subjected to, by their environment. Some of these forces can be exceptionally large, such as the forces on the tendons in the leg of a running elephant, the forces on the elbow of a world-class shot-putter, or the forces on the hide of a water buffalo trying to escape the claws of a lion. It is not surprising that in these situations a tissue can tear or completely fail mechanically. Other forces relevant to living organisms are relatively small but occur frequently, such as deformations of the heart as it beats and the lungs during breathing. At these lower forces we do not expect tears or mechanical failure of tissues. However, so-called fatigue failure of polymers exposed to repetitive stresses over long periods of time is a well-established process. Here, the problem of fatigue failure in proteins such as collagen and elastin is considered.

Taking collagen as an example, the human $\alpha 1$ chain $(\alpha 1(\mathrm{I}))$ collagen subunit is initially synthesized as a 1464 amino acid long tropocollagen molecule (Karsdal, 2019). Tropocollagen is assembled into a helical heterotrimer $\left(\alpha 1(\mathrm{I})_{2}, \alpha 2(\mathrm{I})\right)$ and modified by proteolysis at both ends to produce a collagen molecule. The triple-helical type I collagen molecule is rod-shaped with a length of approximately $300 \mathrm{~nm}$ and a diameter of $1.5 \mathrm{~nm}$. At $300 \mathrm{~nm}$, the individual collagen subunits are extended to $\sim 80 \%$ of their linear contour length. These molecules are then assembled into collagen fibrils, and those fibrils are in turn bundled into collagen fibers.

The coded amino acid sequence for collagen is known from cDNA and genomic sequencing (COL1A1) and is characterized by an especially high frequency of glycine (33\%) and proline $(23 \%)$, organized in repeats of Gly-X-Y, where X or Y is often proline. However, despite such detail, there is significant uncertainty about the actual sequence of amino acids in a collagen molecule in vivo. That uncertainty arises from the significant levels of post-translational modifications of collagen. Two notable modifications are hydroxylation of proline and lysine into 4-hydroxyproline (more rarely 3-hydroxyproline) and 5-hydroxylysine. Typically $\sim 50 \%$ of the 
prolines in collagen are hydroxylated. Approximately $2 \%$ of the amino acids in collagen are lysines, of which $15-90 \%$ are hydroxylated. The extent of hydroxylation of both proline and lysine varies with, for example, age, tissue, and certain pathological conditions. Other modifications include non-enzymatic glycosylation, which is also highly variable. As such, collagen in vivo is a highly heterogenous molecule, and one cannot really speak of a collagen sequence or even some identifiable number of well-defined sequences.

Importantly, collagen molecules are crosslinked intramolecularly, i.e., between collagen monomers within a triple-helical molecule, and crosslinked intermolecularly, i.e., between molecules in a fibril (Yamauchi and Sricholpech, 2012). The crosslinking occurs at hydroxylated lysines that have been glycosylated. Crosslinking plays a central role in determining the mechanical properties of collagenous tissues. It should be noted that post-translational modifications and crosslinking make structural analysis of collagen derived from natural sources difficult. As a result, most of what is known about the atomic structure of collagen arises from the analysis of models based on synthetic peptides (Shoulder and Raines, 2009).

The turnover of collagen in vivo is complicated and depends on the collagen type, the tissue, and the state of collagen maturation (Karsdal, 2019). However, while the details of the turnover of specific collagens in particular situations are poorly understood, it is clear that these molecules turn over very slowly compared to the vast majority of other proteins. Collagen from rat heart and skin are commonly considered to have a half-life of approximately 80 days (Nissen et al., 1978). Laurent has suggested that collagen half-lives are widely overestimated, and that rat heart and skin have half-lives closer to 1-2 weeks (Laurent, 1987). However, even that is very long compared to the half-lives of most proteins (Cambridge et al., 2011).

There is a relative paucity of information about collagen turnover in humans. Babraj et al. (2005) report that based on radioisotope incorporation measurements collagen synthesis in adult human tendon, ligament, muscle, and skin is of order $0.04 \% / \mathrm{hr}$, which at steady state corresponds to an average half-life of $\sim 70$ days. However, these measurements do not directly address the halflife of collagen. Using atmospheric $\mathrm{C}^{14}$ prevalence from nuclear weapons testing, Heinemeier et al. (2013) concluded that after the first 17 years of life, human Achilles tendon collagen "is essentially not renewed." These observations can likely, at least in part, be reconciled with the measurement by Babraj and coworkers by assuming that some parts of tendons turnover quickly, while there is also a highly stable core. Measurements using amino acid racemization suggest that collagen in human skin has a half-life of $\sim 15$ years and the collagen in cartilage has a half-life of $>100$ years (Verzijl et al., 2000). Similar measurements for vertebral discs show a collagen halflife of 100-200 years, depending on age (Sivan et al., 2008).

Both natural and synthetic elastomers are widely used in products, ranging from car tires to gloves to artificial vascular grafts. These types of polymers have been extensively studied to optimize their performance and establish limits for their use. For elastomers, one significant mode of failure is referred to as fatigue (Bolotin, 1998). Fatigue failure arises in materials that are repeatedly, often cyclically, stressed, which over time forms a crack that grows to a point that the structure fails. The crack may nucleate at a pre-existing defect in the polymer or at a site of bond breakage that arises from use and propagate via polymer chain scission. Not surprisingly, fatigue failure depends on the rate and magnitude of the deformation. At sufficiently high forces and deformations, a polymer will fail instantaneously. However, at relatively small deformations, common elastomers will often last many millions of stress-strain cycles before failing. For example, Abraham et al. (2001) showed that a standard formulation of the widely used ethylene propylene diene monomer (EPDM) rubber, at strains of $\sim 5 \%$ and deformation rates of $1 \mathrm{~Hz}$, fails 
at slightly less than 1 million cycles. Flamm and coworkers (2008) reported a large scatter, from $\sim 100,000$ to several million, of cycles-to-failure for EPDM. They also reported the failure of natural rubber, for low strains, at a few million cycles. Because initial defect formation precedes failure, these measures of fatigue failure are an upper bound for the failure of individual chemical bonds in polymers.

Collagenous tissues have elastomeric properties, which is to say that when deformed, they can passively return to their original shape. At 70 beats per minute, the human heart and the associated vasculature undergo nearly 40 million deformation cycles per year. Given the long half-life of collagen, this could mean that some molecules undergo many hundreds of millions of cycles. There is limited data on what would be considered fatigue failure of biological tissues. The standard for determining fatigue failure is the Wöhler curve ( $\mathrm{S}-\mathrm{N}$ curve), in which the number of cycles to failure is plotted for a range of stresses (or strains) applied to the sample (Bolotin, 1999). These curves can be viewed as characterizing the mechanical capacity of a material. Belluci and Seedhom (2002) found tensile fatigue failure for knee cartilage around 1-2 million cycles of deformation for a range of parameters. Riemenschneider et al. (2019) studied compressive fatigue in the patellar tendon and found that at strains of 50-80\%, samples typically failed around a few hundred cycles. At $30-40 \%$ strains many samples failed around 10,000 cycles, although some went beyond the 100,000 -cycle limit of the study. Extrapolation of the data suggests that strains of $25 \%$ would result in failure at about $\sim 1,000,000$ cycles. While similar data does not appear to be available for the heart, strains of several tens of percent are well within the range involved in a typical heartbeat (Voigt et al., 2019).

If molecules such as collagen are breaking during important biological functions, such as the beating of the heart, that prompts the question of how and where in the molecule does such a protein break? What bonds in mechanically stressed proteins are most likely to break, and what are the consequences of bond breaking? To gain insight into this problem the relative strengths of bonds along the backbone of a protein are estimated using bond dissociation energies (BDEs). Model compounds, for which BDE's are available (Luo, 2007), are used to estimate protein backbone bond strengths. These models were selected to be structurally similar to the backbone bonds. To the extent possible, the bonds adjacent to the bond in question are of the same type and are connected to the same atom type. Using that approach, there are three classes of amino acids that need to be represented. Glycine, in which the R-group bonded to the backbone is hydrogen, proline which is composed of a pyrrolidine ring that forms part of the backbone, and alanine through which a methyl group is connected to the backbone. Alanine serves as a model for all other amino acids, since all other side chains are connected to the backbone via an SP3 bonded carbon.

\section{$\underline{\text { Results }}$}

A convenient and relevant model peptide based on the three amino acid types is GPAG, which also occurs naturally in collagen (Figure 1). The model-based BDEs for the bonds along the GPAG backbone range from $302 \mathrm{~kJ} / \mathrm{mole}$ to $422 \mathrm{~kJ} / \mathrm{mol}$ (Table 1). The model compounds for proline do not account for ring strain in the pyrrolidine ring. That ring strain is here estimated from the difference between cyclopentane and cyclohexane to be $31 \mathrm{~kJ} / \mathrm{mol}$ (Khoury et al., 2004). Therefore, the N-Ca bond (position 3-4) in the proline is adjusted from $302 \mathrm{~kJ} / \mathrm{mol}$ to $271 \mathrm{~kJ} / \mathrm{mol}$. The N-C $\alpha$ for both glycine and alanine are based on ethyl methylamine $(334 \mathrm{~kJ} / \mathrm{mol})$. As a result, the $\mathrm{N}-\mathrm{C} \alpha$ in proline is $>60 \mathrm{~kJ} / \mathrm{mol}$ weaker than the next weakest bond in the backbone. However, if the BDE was available, N-methylisopropylamine would be a better model for the alanine N-C $\alpha$ 
bond. That would likely be slightly smaller BDE than ethyl methylamine, and thus the difference between proline and the next weakest bond is probably an overestimate. It should be noted that C$\mathrm{N}$ is the peptide bond, which has a partial double bond character that makes it stronger and gives it a planar geometry. Note also that backbone bonds for all other amino acids would be based on the same models as alanine.

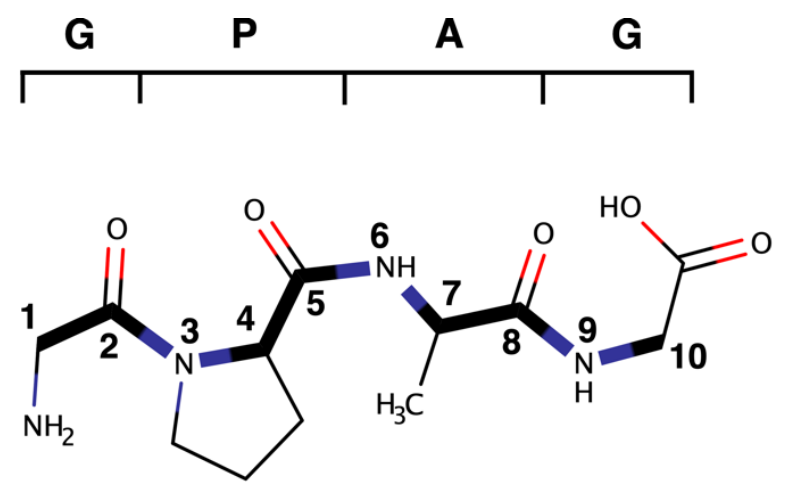

Figure 1. Structure of the peptide GPAG used to estimate peptide backbone bond strengths. The atoms at positions $1,4,7$, and 10 are the $\alpha$ carbons of the respective amino acids. The bottom structure shows the BDEs $(\mathrm{kJ} / \mathrm{mol})$ of the bonds along the backbone (from Table 1). The strongest are the peptide bonds (e.g. 5-6), and the weakest is the N-C $\alpha$ bond of proline (34).

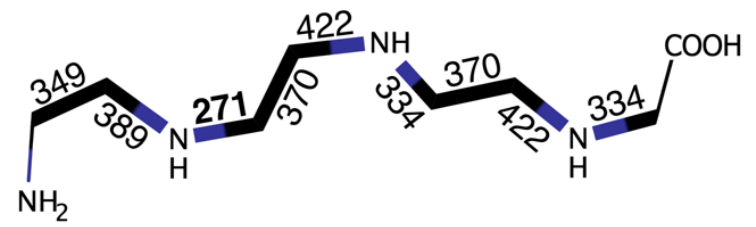

\begin{tabular}{|l|l|l|l|l|}
\hline & Position & Model & Bond & $\begin{array}{l}\text { BDE } \\
\text { (kJ/mol }\end{array}$ \\
\hline \multirow{2}{*}{ Glycine } & $1-2$ & Amino Acetic Acid & $\mathrm{NH}_{2} \mathrm{CH}_{2}-\mathrm{C}(\mathrm{O}) \mathrm{OH}$ & 349 \\
\cline { 2 - 5 } & $2-3$ & N,N-Dimethyl Acetamide & $\mathrm{CH}_{3} \mathrm{C}(\mathrm{O})-\mathrm{N}\left(\mathrm{CH}_{3}\right)_{2}$ & 382 \\
\hline \multirow{3}{*}{ Proline } & $3-4$ & Ethyl Dimethylamine & $\mathrm{N}\left(\mathrm{CH}_{3}\right)_{2}-\mathrm{C}_{2} \mathrm{H}_{5}$ & $302,271^{*}$ \\
\cline { 2 - 5 } & $4-5$ & Acetamide & $\mathrm{CH}_{3}-\mathrm{C}(\mathrm{O}) \mathrm{NH}_{2}$ & 370 \\
\cline { 2 - 5 } & $5-6$ & N-Methyl Acetamide & $\mathrm{CH}_{3} \mathrm{C}(\mathrm{O})-\mathrm{NHCH}_{3}$ & 422 \\
\hline \multirow{3}{*}{ Alanine } & $6-7$ & Ethyl Methylamine & $\mathrm{C}_{2} \mathrm{H}_{5}-\mathrm{NHCH}_{3}$ & 334 \\
\cline { 2 - 5 } & $7-8$ & Acetamide & $\mathrm{CH}_{3}-\mathrm{C}(\mathrm{O}) \mathrm{NH}_{2}$ & 370 \\
\cline { 2 - 5 } & $8-9$ & N-Methyl Acetamide & $\mathrm{CH}_{3} \mathrm{C}(\mathrm{O})-\mathrm{NHCH}_{3}$ & 422 \\
\hline Glycine & $9-10$ & Ethyl Methylamine & $\mathrm{C}_{2} \mathrm{H}_{5}-\mathrm{NHCH} \mathrm{H}_{3}$ & 334 \\
\hline
\end{tabular}

Table 1. Model compounds used for estimating the bond dissociation energies for the backbone of the GPAG peptide (data from Luo, 2007). The compounds are chosen so that the moieties adjacent to the bond in question are chemically as similar as possible to the relevant amino acid. The numbering of the position of atoms is shown in Figure 1. *The BDE for ethyl dimethylamine of $302 \mathrm{~kJ} / \mathrm{mol}$ is adjusted by subtracting $31 \mathrm{~kJ} / \mathrm{mol}$ to account for strain in the pyrrolidine ring.

A limitation of using BDEs to estimate bond strength is that these ignore the possibility of chemical reactions involving the stressed bonds. Stress-induced changes to chemical reactivity are well-known and could in principle favor bond breakage at other positions in the backbone (Beyer and Clausen-Schaumann, 2005). Although, changes in chemical reactivity could also make proline ring-opening even more probable relative to other bonds. 
This analysis suggests that prolines in proteins that are subjected to significant, often cyclical, mechanical stresses, such as those in type I collagen, will tend to break at the $\mathrm{N}-\mathrm{C} \alpha$ bond before other bonds in the backbone of the polypeptide chain. In doing so, the pyrrolidine ring of proline opens into a linear molecule, which maintains the covalent continuity of the collagen backbone (Figure 2). This open-proline-bridge (OP-bridge) thereby reduces the frequency of backbone scission events. The opening of the pyrrolidine ring also has the effect of reducing the local stiffness of the collagen molecule, thereby redistributing applied forces. Thus, mechanically induced proline ring-opening provides a mechanism to protect the covalent integrity collagens, as well as to remodel the mechanical properties of collagenous structures over time.

A

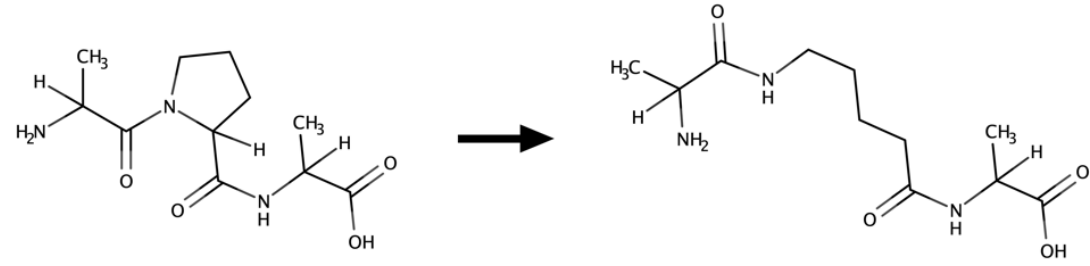

B
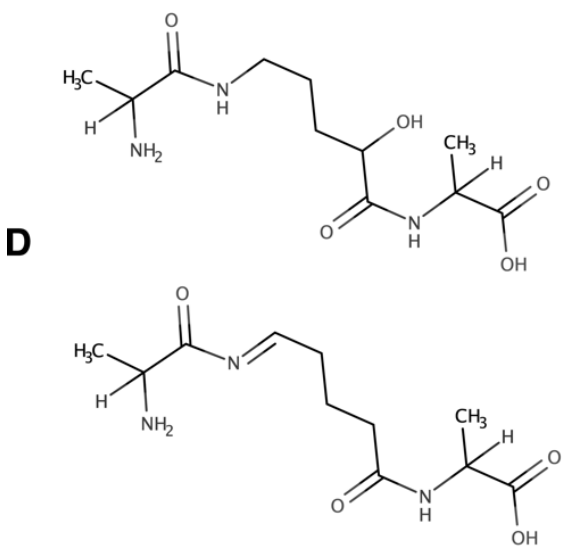

C
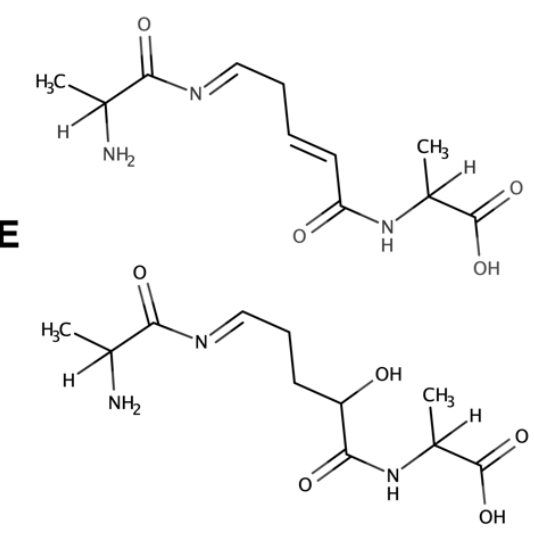

Figure 2. A. The proposed mechanically promoted ring-opening of proline to produce an OPbridge with 5-aminovaleric acid inserted along the peptide backbone, in a GAPG peptide. B-E. Potential alternate ring-opening products and OP-bridges.

The simplest product of a proline ring-opening is 5-aminovaleric acid (AVA), forming an OPbridge that connects adjacent amino acids. Notably, AVA is already commercially available as a spacer in peptide synthesis (e.g., Pepscan, Lelystad, The Netherlands). However, the ring-opening likely involves the formation of free radicals (Beyer and Clausen-Schaumann, 2005), which in the presence of water could produce a number of other structures (e.g., Figure 2 B-E). A simple ringopening to form AVA increases the distance between atom 3 and atom 4 in GPAG from $\sim 1.5 \AA$ to $\sim 4.5 \AA$. Given the large number of prolines, this could in principle increase the end-to-end length of a collagen monomer by $73 \mathrm{~nm}$. However, more likely only a small fraction of prolines in a collagen molecule ever undergo ring-opening. OP-bridges would also form for the hydroxylated prolines, 4-hydroxyproline or 3-hydroxyproline, which in the simplest cases would form 4hydroxy 5-aminovaleric acid and 3-hydroxy 5-aminovaleric acid OP-bridges. These bridges may also have structures similar to the alternates for AVA. 
The relative stability of the proline N-C $\alpha$ bond to the next weakest bond (e.g., the N-C $\alpha$ bond of alanine) can be approximated by using the difference in BDEs (60 kJ/mol or $14.6 \mathrm{kT} / \mathrm{molecule})$. Making the simplifying assumption that the only difference between the bonds is the BDE, the Bell model (Bell, 1978) can be used to estimate that the proline ring-opening is $\sim 2$ million times more probable than the breaking of the N-Ca bond of alanine in the GPAG peptide.

\section{Discussion}

Proline is the weakest link

The main finding of the analysis performed here is that proline, specifically N-C $\alpha$ bond of proline, is mechanically weaker than any other bond in the peptide backbone. The immediate consequence of this finding is that proline ring-opening maintains covalent continuity of the protein backbone, and thereby protects proteins against mechanically induced backbone scission. Further, a mechanically enhanced breaking of the N-C $\alpha$ bond modifies the mechanical properties of proteins and reduces the probability of mechanically induced damage in tissues. The probability of bond scission in a polymer undergoing cyclic stresses depends on the number of cycles of deformation. Because of this, a relatively simple prediction of this model is that there is a gradual increase of OP-bridges in the tissues of some organs, such as the heart and lung, over time. Although it is also possible that OP-bridges form rarely during normal tissue function and only become important in situations of diseases or unusual patterns of mechanical stress. In that case, the situations in which OP-bridges form still need to be identified.

\section{A proline clock?}

Given the basic relationship between stress-strain cycles and bond breakage noted above, one interesting possibility is that the conversion of prolines to OP-bridges acts as a clock of sorts. There are likely many different molecules that employ the OP-bridge, and these reside in different tissues. As a result, there would be many proline clocks running in parallel, which could provide a new mechanical view on the development and maturation of tissues in many organisms. Further, peptides derived from molecules such as collagen and elastin are found in the circulatory system, and a simple blood test might provide information on the mechanical age of the tissues from which they are derived.

\section{Sacrificial bonds}

If proteins such as collagen have evolved to include prolines, at least in part because of the mechanical benefits provided by the proline ring-opening, then the proline $\mathrm{N}-\mathrm{C} \alpha$ bond can be viewed as a sacrificial bond. That is to say, the proline $\mathrm{N}-\mathrm{C} \alpha$ is part of the structure so that it can be sacrificed to protect the integrity of the peptide backbone. It is worth noting that the term "sacrificial bond" is used with respect to the mechanical properties of polymers in at least two other contexts. Hansma and colleagues (Fantner et al., 2005) have shown certain bonds in a polymer, if subjected to tension, can break in a way so as to release so-called hidden length of the polymer. The release of the hidden length effectively resets the polymer spring, thereby increasing the amount of energy required to separate two points attached to the polymer. The term sacrificial bond has also been used to describe bonds in elastomers that improve mechanical properties by dissipating energy upon bond breaking (Webber et al., 2007; Ducrot et al., 2014). In principle, the proline ring-opening has elements of both mechanisms, although it seems unlikely that either plays a significant role in the present context. Further, neither captures the primary importance of the 
proline ring-opening, which is that sacrificing the $\mathrm{N}-\mathrm{C} \alpha$ proline bond redistributes forces, while at the same time protecting the integrity of the peptide backbone.

Free radicals from bond breaking in collagen

One study that deserves particular attention here is the investigation of mechanically induced bond breaking in tendon by Gräter and colleagues (Zapp et al., 2020). These authors concern themselves primarily with the problem of oxidative stress and the role that collagen can play as a source or sink of free radicals. They demonstrate that mechanically stressed tendons produce free radicals, for which bond-breaking seems the only reasonable explanation. Notably, the authors specifically address the bonds that might break during applied stress. To quote directly from the relevant section, "Taken together, crosslinks and the backbone in their direct vicinity are most stressed. Therein, the candidates for bond scission are the single bonds, which are of either $\mathrm{C}-\mathrm{C}$ or $\mathrm{C}-\mathrm{N}$ type. These two bond types exhibit dissociation energies in the same range, 352-377 $\mathrm{kJ} / \mathrm{mol}^{26}$. CASPT2 calculations of representative crosslink and backbone fragments yielded 352$355 \mathrm{~kJ} / \mathrm{mol}$, and confirmed that both $\mathrm{C}-\mathrm{C}$ and $\mathrm{C}-\mathrm{N}$ bonds are candidates for mechanoradical formation by homolytic bond scission (Supplementary Fig. 2e)." While the bond energies used by Zapp et al. are from the same source as those used in the present work (Luo, 2007, their reference 26), they do not specify the molecules from which their energies are drawn. As a result, a direct comparison with the present work is not possible. It should be noted that they made no distinction between any of the $\mathrm{C}-\mathrm{N}$ bonds and the $\mathrm{C}-\mathrm{C}$ bonds, and do not appear to account for contributions from the carbonyl group in the peptide bond.

One important aspect of the picture developed by Zapp et al. is that they use the results of a simulation to conclude that forces on a collagen fibril are larger in the vicinity of cross-links. The "vicinity" appears to include residues within 10 amino acids of a crosslink. In collagen I, there are 9 lysines with a proline immediately adjacent, and every lysine has a proline within 10 residues. Thus, prolines would still be more prone to ring-opening than the breaking of other bonds in the peptide backbone.

\section{Mechanical remodeling}

Beyond protecting proteins from scission of the peptide backbone, proline ring-opening could also allow for a mechanical remodeling of tissues. In the simplest case, one assumes that there are naturally occurring inhomogeneities in force propagation through collagen fibrils that arise during tissue formation. In that case, the molecules supporting larger forces will undergo ring-opening more quickly than others, causing a redistribution of forces to make the forces more evenly distributed (Figure 3). Growth of tissues could also cause changes in how stresses are distributed, and proline ring-opening could provide a mechanism to accommodate such changes against a background of slow protein turnover.

Ring-opening could also play a role in localized tissue injury. For example, the interruption of blood flow to a part of the heart associated with a heart attack can cause localized loss of muscle function. That loss of contractility, in turn, causes changes to how a heart moves and could give rise to a significant redistribution of how forces are propagated. In this case, ring-opening would serve to compensate for the mechanical changes that occur as a result of the injury or disease. Parts of the tissue where forces increase would experience an increase in $\mathrm{N}-\mathrm{C} \alpha$ bond breaking, and these would act to reduce the frequency of peptide backbone scission. 


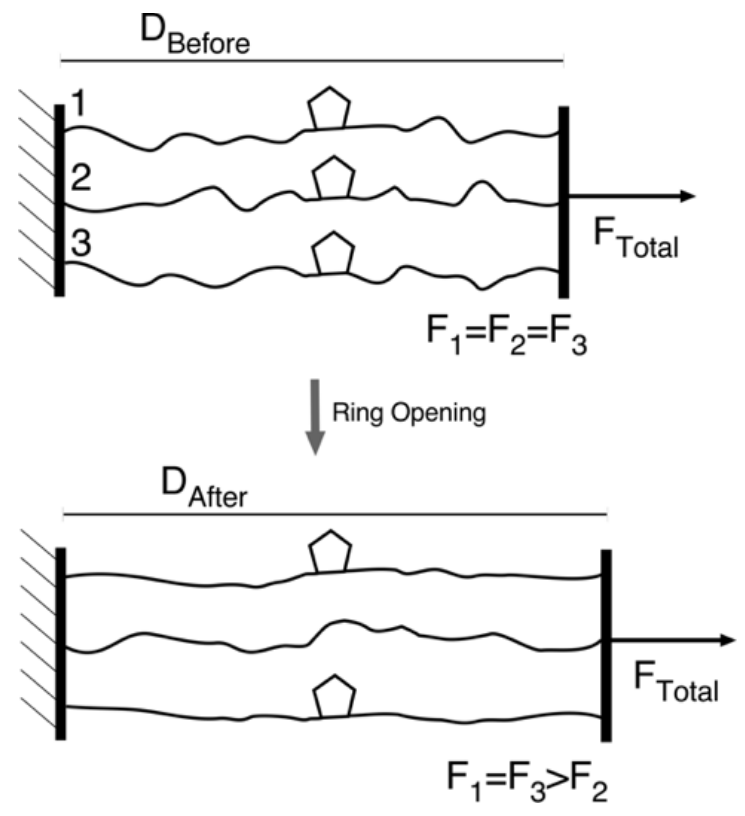

Figure 3. Highly simplified model illustrating how a ring-opening would redistribute forces between nearby molecules. The model is composed of three identical polymers (1-3) with a ring structure in the middle. The polymers are all immobilized on the left and anchored to a movable surface to the right. A force $\left(\mathrm{F}_{\text {Total }}\right)$ applied to the right side extends the polymers some distance ( $\left.\mathrm{D}_{\text {Before }}\right)$. For the case in which all three polymers are identical, the total force is evenly distributed among them $\left(\mathrm{F}_{1}=\mathrm{F}_{2}=\mathrm{F}_{3}\right)$. A ring-opening in polymer 2 extends the length of that polymer, reducing the stiffness of the molecule. Without a change in total force, D then increases to $\mathrm{D}_{\mathrm{after}}$, and the force distribution changes so that $\mathrm{F}_{1}=\mathrm{F}_{3}>\mathrm{F}_{2}$.

\section{Elastin and other proteins}

Elastin is another example of a protein in which proline ring-opening may play a functional role. Elastin plays important mechanical roles in, for example, the lung and the vasculature, where it imparts elastic properties. Elastin is a long-lived protein, and in the human lung, it can have a half-life of over 70 years (Shapiro et al., 1991). Given a breathing frequency of about 17 breaths per minute, an elastin molecule would be subjected to $\sim 9$ million stress-strain cycles per year. Mature elastin is a complicated structure based on a network of cross-linked elastin monomers (Mithieux and Weiss, 2005). Elastin is particularly rich in glycine (29\%), alanine (21\%), valine $(13 \%)$, and proline (13\%). The molecule is composed of alternating hydrophilic and hydrophobic domains. The hydrophobic domains are characterized by repeats similar to GVGVP, GGVP, or GVAP, while the hydrophilic domains are rich in lysine and alanine. Elastin monomers have a relatively globular structure, and elasticity arises from the extension of the molecules ( $\mathrm{Li}$ and Daggett, 2002). In situations when some parts of the elastin network are subject to more stress than others, ring-opening of prolines would tend to redistribute forces, while reducing the frequency of polypeptide backbone scission.

Interestingly, it has been shown that elastin-derived peptides with the GXXPG motif induce an emphysema-like response in mice (Sellami et al., 2016). That response was also shown to require a carboxyterminal glycine on the peptide. In the present context, that raises the question of how the peptide backbone of a protein such as elastin or collagen breaks, and what are the structures of the new ends when it does? Might the peptide backbone scission of elastin product expose a new end that would bind to an elastin binding protein and activate responses similar to the free peptide from the corresponding part of the molecule? More broadly, how do tissues normally respond to the breaking of tension-carrying molecules?

Titin is yet another protein that could benefit from mechanically induced ring-opening of proline. Titin is subjected to cyclic (heart) and non-cyclic (skeletal muscle) stresses that could lead to fatigue failure. The passive elasticity of titin arises predominantly from the extension of PEVK domains (Linke et al., 1998). In the heart, the stress-strain cycles would follow those of collagen as discussed above. In skeletal muscle, the stress-strain cycles would be less regular, though for many muscles, still quite frequent — while, for example, walking. 
Ring-opening in synthetic polymers

The effect of forces on the chemistry of ring-opening has been studied in several systems (Caruso et al., 2009). One of the primary effects of an applied force is the increase in reaction rates. For example, forces applied to benzocyclobutene will increase the rate of opening of the cyclobutene ring. Thus, benzocyclobutenes incorporated into a polymer such as polyethylene glycol undergo a ring-opening that depends on the stress applied to the polymer. A similar effect can be for spiropyran incorporated into a polymer, where mechanical stress on a polymer can induce a ring-opening to produce its merocyanine form. The focus of the present work is on the the strength of bonds in the peptide backbone, and mechanically induced ring-opening of proline that improves the mechanical performance of a tissue. But it is not difficult to extend this idea to ring-based structures in other polymers.

EPDM rubber is a widely used industrial polymer, of which there are millions of tons produced each year. EPDM is produced by copolymerization of ethylene, propylene, and a diene, such as ethylidene norbornene, at varying ratios. The result is a highly heterogeneous polymer, which is often represented in average or idealized structure (Figure 4). In the context of the proline ringopening model, it is interesting to note that EPDM contains a bicyclic ring structure that lies in the backbone of the polymer. The $\mathrm{C}-\mathrm{C}$ bond along the polymer backbone, the in-backbone bond, is shared with a five-membered ring that is fused with another five-membered ring. This structure would appear to produce a strain on the in backbone bond similar to cyclopentane alone (Khoury et al., 2004). Breaking of the in-backbone $\mathrm{C}-\mathrm{C}$ bond would release $\sim 3 \AA$ of additional length and leave a five-membered ring in the backbone. The remaining ring could subsequently also open to provide additional mechanical relief. Thus, ring-opening could serve to protect the polymer backbone and reduce the nucleation of fatigue cracking.

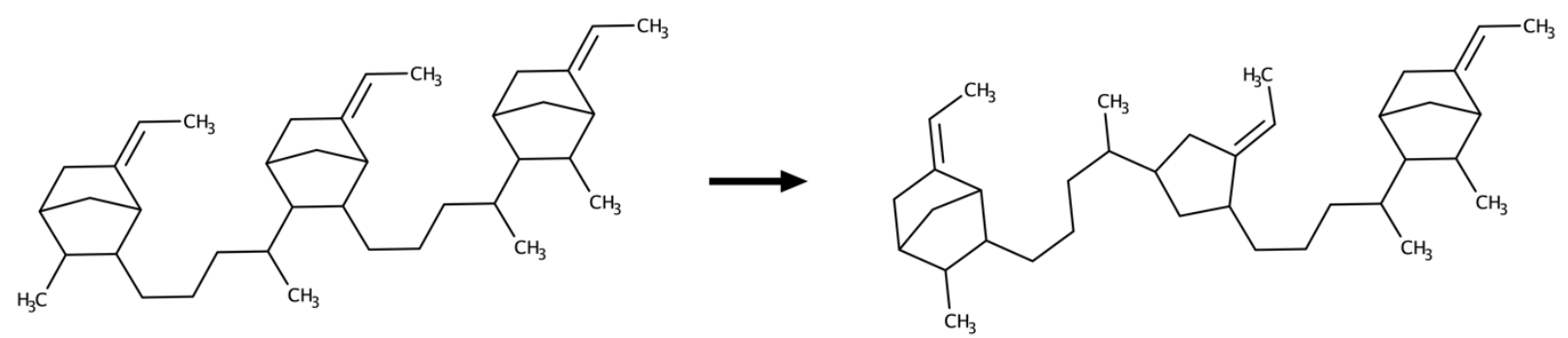

Figure 4. Potential ring-opening in EPDM. EPDM rubber is a heteropolymer with a highly heterogeneous structure and is here shown it is typically illustrated. Breaking of the C-C bond of the bicyclic bridge that lies in the polymer backbone would increase the polymer chain length, leave a five-membered ring in the backbone, and maintain the covalent continuity of the backbone.

There are other industrial polymers in which a cyclic alkane lies in the polymer backbone. Taking into consideration the mechanical effects of ring-opening may offer a design element for synthetic polymers, wherein ring strain can be controlled and bond strengths can be modified with substituents on adjacent atoms. For example, cycloheptane has a ring strain very close to that of cyclopentane, but the opening of a cycloheptane-based ring would increase the distance between two adjacent carbons from $1.5 \AA$ to $7.3 \AA$. That would relieve more stress on the polymer chain than the opening of a five-membered ring. 


\section{Concluding remarks}

One might wonder why an OP-bridge modification has not yet been reported experimentally, particularly given the ubiquity of mass spectrometric analysis of proteins. To begin, the mass differences are small and an AVA-based OP-bridge is only two Dalton heavier than the proline ring from which it is derived. Some of the prolines, including those with an open ring, are also hydroxylated and there are a number of possible open forms. Further, elastin and collagen are highly cross-linked and undergo enzyme-independent glycosylation over time. Thus, the mass spectra of these molecules are extremely complicated. Prolines with open rings also likely represent a small fraction of the total number of prolines. Taken together, it does not seem unreasonable that the OP-bridge has avoided detection to date.

\section{Acknowledgment}

The train of thought that led to the present work started with an experiment observation by Dr. Maria Hoh, who noticed some surprising features in micropatterned substrates collagen. The search for an understanding of those features eventually lead to the present report.

\section{Address for correspondence}

Jan Hoh, 6644 Finley Place, \#201, Boulder, CO 80301

\section{References}

Abraham, F., Alshuth, T., Jerrams, S., 2001. Dependence on mean stress and stress amplitude of fatigue life of EPDM elastomers. Plastics, Rubber and Composites 30, 421-425.

Babraj, J.A., Cuthbertson, D.J.R., Smith, K., Langberg, H., Miller, B., Krogsgaard, M.R., Kjaer, M., Rennie, M.J., 2005. Collagen synthesis in human musculoskeletal tissues and skin. Am. J. Physiol-Endocrin. Metab. 289, E864-E869.

Bell, G.I., 1978. Models for the Specific Adhesion of Cells to Cells. Science 200, 618-27.

Bellucci, G., Seedhom, B.B., 2002. Tensile fatigue behaviour of articular cartilage. Biorheology 39, 193-199.

Beyer, M.K., Clausen-Schaumann, H., 2005. Mechanochemistry: The Mechanical Activation of Covalent Bonds. Chem. Rev. 105, 2921-2948.

Bolotin, V.V., 1999. Mechanics of Fatigue. Taylor \& Francis Group, Boca Raton.

Cambridge, S.B., Gnad, F., Nguyen, C., Bermejo, J.L., Krüger, M., Mann, M., 2011. Systemswide proteomic analysis in mammalian cells reveals conserved, functional protein turnover. J. Proteome Res. 10, 5275-5284.

Caruso, M.M., Davis, D.A., Shen, Q., Odom, S.A., Sottos, N.R., White, S.R., Moore, J.S., 2009. Mechanically-Induced Chemical Changes in Polymeric Materials. Chem. Rev. 109, 5755-5798. 
Ducrot, E., Chen, Y., Bulters, M., Sijbesma, R.P., Creton, C., 2014. Toughening elastomers with sacrificial bonds and watching them break. Science 344, 186-189.

Fantner, G.E., Hassenkam, T., Kindt, J.H., Weaver, J.C., Birkedal, H., Pechenik, L., Cutroni, J.A., Cidade, G.A.G., Stucky, G.D., Morse, D.E., Hansma, P.K., 2005. Sacrificial bonds and hidden length dissipate energy as mineralized fibrils separate during bone fracture. Nat Mater 4, 612-616.

Flamm, M., Steinweger, T., Spreckels, J., Brüger, T., 2008. Mechanical properties of EPDM. Presented at the ECCMR V, pp. 233-238.

Heinemeier, K.M., Schjerling, P., Heinemeier, J., Magnusson, S.P., Kjaer, M., 2013. Lack of tissue renewal in human adult Achilles tendon is revealed by nuclear bomb (14)C. FASEB J 27, 20742079.

Karsdal, M.A., 2019. Biochemistry of Collagens, Laminins and Elastin, 2nd ed. Academic Press, London.

Khoury, P.R., Goddard, J.D., Tam, W., 2004. Ring strain energies: substituted rings, norbornanes, norbornenes and norbornadienes. Tetrahedron 60, 8103-8112.

Laurent, G.J., 1987. Dynamic state of collagen: pathways of collagen degradation in vivo and their possible role in regulation of collagen mass. Am. J. Physiol. 252, C1-9.

Li, B., Daggett, V., 2002. Molecular basis for the extensibility of elastin. J Muscle Res Cell Motil 23, 561-573.

Linke, W.A., Ivemeyer, M., Mundel, P., Stockmeier, M.R., Kolmerer, B., 1998. Nature of PEVKtitin elasticity in skeletal muscle. Proc. Natl. Acad. Sci. U.S.A. 95, 8052-8057.

Luo, Y.R., 2007. Handbook of chemical bond energies. CRC Press, Boca Raton.

Mithieux, S.M., Weiss, A.S., 2005. Elastin. Adv. Protein Chem. 70, 437-461.

Nissen, R., Cardinale, G.J., Udenfriend, S., 1978. Increased Turnover of Arterial Collagen in Hypertensive Rats. Proc. Nat. Acad. Sci. U.S.A. 75, 451-453.

Riemenschneider, P.E., Rose, M.D., Giordani, M., McNary, S.M., 2019. Compressive fatigue and endurance of juvenile bovine articular cartilage explants. J. Biomechan. 95, 109304.

Sellami, M., Meghraoui-Kheddar, A., Terryn, C., Fichel, C., Bouland, N., Diebold, M.-D., Guenounou, M., Héry-Huynh, S., Le Naour, R., 2016. Induction and regulation of murine emphysema by elastin peptides. Am. J. Physiol. Lung. Cell. Mol. Physiol. 310, L8-23.

Shapiro, S.D., Endicott, S.K., Province, M.A., Pierce, J.A., Campbell, E.J., 1991. Marked longevity of human lung parenchymal elastic fibers deduced from prevalence of D-aspartate and nuclear weapons-related radiocarbon. J. Clin. Invest. 87, 1828-1834. 
Shoulders, M.D., Raines, R.T., 2009. Collagen Structure and Stability. Annu. Rev. Biochem. 78, 929-958.

Sivan, S.-S., Wachtel, E., Tsitron, E., Sakkee, N., van der Ham, F., DeGroot, J., Roberts, S., Maroudas, A., 2008. Collagen Turnover in Normal and Degenerate Human Intervertebral Discs as Determined by the Racemization of Aspartic Acid. J. Biol. Chem. 283, 8796-8801.

Verzijl, N., DeGroot, J., Thorpe, S.R., Bank, R.A., Shaw, J.N., Lyons, T.J., Bijlsma, J.W.J., Lafeber, F.P.J.G., Baynes, J.W., TeKoppele, J.M., 2000. Effect of Collagen Turnover on the Accumulation of Advanced Glycation End Products*. J. Biol. Chem. 275, 39027-39031.

Voigt, J.-U., Cvijic, M., 2019. 2- and 3-Dimensional Myocardial Strain in Cardiac Health and Disease. JACC: Cardiovasc. Imag. 12, 1849-1863.

Webber, R.E., Creton, C., Brown, H.R., Gong, J.P., 2007. Large Strain Hysteresis and Mullins Effect of Tough Double-Network Hydrogels. Macromolecules 40, 2919-2927.

Yamauchi, M., Sricholpech, M., 2012. Lysine post-translational modifications of collagen. Essays Biochem. 52, 113-133. 